\title{
Singular shell embedded into a cosmological model
}

\author{
Øyvind Grøn* \\ Oslo College, Faculty of Engineering and \\ Dep. of Physics, University of Oslo \\ Peter D. Rippis ${ }^{\dagger}$ \\ Dep. of Physics, University of Oslo
}

July 5, 2018

\begin{abstract}
We generalize Israel's formalism to cover singular shells embedded in a non-vacuum Universe. That is, we deduce the relativistic equation of motion for a thin shell embedded in a Schwarzschild/FriedmannLemaître-Robertson-Walker spacetime. Also, we review the embedding of a Schwarszchild mass into a cosmological model using "curvature" coordinates and give solutions with (Sch/FLRW) and without the embedded mass (FLRW).
\end{abstract}

\section{Introduction}

The first applications of Israel's relativistic theory of singular surfaces [1, 2] were concerned with neutral and charged surfaces layers in static spacetimes with or without a cosmological constant [3] - 7]. Surface layers with different equations of state, i.e. different relationships between pressure and density were considered, for example domain walls 8. Also, methods to quantize a shell in general relativity has been developed and studied for static spacetimes 9 [10. Lately Israel's theory has been applied to three-dimensional branes in a fivedimensional bulk [11, 12.

In the present work we want to extend the application of Israel's formalism to cases with singular layers (two-dimensional branes) in expanding Universe models. In the static case with a neutral surface layer there is Schwarzschild or Schwarzschild-de Sitter spacetime. For non-static spacetimes the relativistic equation of motion for surface layers has been given for Friedmann-LemaitreRobertson-Walker (FLRW) spacetimes [13] - [16]. In the more general case we must consider spacetime outside a mass embedded in an expanding Universe model. A description of this was developed by R. Gautreau [17, 18].

\footnotetext{
*e-mail: Oyvind.Gron@iu.hio.no

${ }^{\dagger}$ e-mail: p.d.rippis@fys.uio.no
} 
We shall therefore apply Israel's theory to a singular layer with the Gautreau metric outside the shell. The equation of motion for the general case is deduced here.

A similar problem, with a slowly rotating shell in an almost FLRW dust universe model was considerd by Klein [19] without using Israel's formalism and later by T. Doležel, J. Bičák and N. Deruelle 20] using Israel's formalism. They restricted their treatments by considering only a shell comoving with the cosmic dust outside the shell, and with Minkowski spacetime inside the shell. In our treatment we consider a non-rotating shell that needs not be comoving with the cosmic fluid.

\section{Schwarzschild mass embedded into a cosmo- logical model}

Embedding a Schwarzschild mass, $m$, into a cosmological model is most easily done in "curvature" coordinates. That is, in coordinates for which the radial coordinate $R$ gives an angular part $R^{2} d \Omega^{2}$. The embedding of a Schwarzschild mass into a spatially flat FLRW Universe model was given by R. Gautreau in 17. Also, a thorough investigation of the FLRW models with vanishing cosmological constant, $\Lambda=0$, in these coordinates has been performed by $\mathrm{R}$. Gautreau, see [21] and references therein. In this section we give a short review. Then we give the equations for embedding a Schwarzschild mass into a general FLRW Universe model and provide solutions with and without the embedded mass.

\section{$2.1 \quad$ The metric}

For a spherically symmetric spacetime we can write a general metric as:

$$
d s^{2}=-A(R, T) f^{2}(R, T) d T^{2}+\frac{d R^{2}}{A(R, T)}+R^{2} d \Omega^{2},
$$

where

$$
d \Omega^{2}=d \theta^{2}+\sin ^{2} \theta d \phi^{2}
$$

Here $A$ and $f$ are functions of $T$ and $R$ that are settled by Einstein's field equations. The physical interpretation of the time coordinate $T$ is that it measures the time on clocks that are located at points for which $R=$ constant relative to our chosen origin $R=0 .{ }^{1}$ In the FLRW Universe models one can define a global time coordinate. Hence, we wish to record time on a geodesically moving clock. To transform to a geodesically moving clock we consider the geodesic equation, $V_{; \nu}^{\mu} V^{\nu}=0$, for a radially moving clock, i.e $d \theta=d \phi=0$; where $V^{\mu}$ is the tangent vector to the geodesic curve. For timelike geodesics, $V^{\mu} V_{\mu}=-1{ }^{2}$ the solution is found to be (see [17]):

\footnotetext{
${ }^{1}$ The time laps in $\mathrm{T}$ and the time laps recorded on clocks that measures proper time $\tau$ $\left(d s^{2}=-d \tau^{2}\right)$ for $R, \theta, \phi=$ constant are related by $d T=\frac{1}{f \sqrt{A}} d \tau$.

${ }^{2}$ We use units where the speed of light and the gravitational constant are set to unity, $c=G=1$.
} 


$$
V^{\mu}(T, R) \equiv \frac{d x^{\mu}}{d \tau}=\left(\frac{\mathcal{E}}{f A}, \varsigma\left(\mathcal{E}^{2}-A\right)^{\frac{1}{2}}, 0,0\right), \varsigma= \pm 1,
$$

with the conditions (coordinate transformations):

$$
\begin{aligned}
t_{, \mathrm{R}} & =-\varsigma A^{-1}\left(\mathcal{E}^{2}-A\right)^{\frac{1}{2}} \\
t_{, \mathrm{T}} & =\mathcal{E} f,
\end{aligned}
$$

where $\mathcal{E}$ is an energy parameter for the reference particles, i.e. the geodesic clocks. $\mathcal{E}$ depends on our choice of reference system, and can be used to describe the open, closed and flat FLRW Universe models. The time coordinate $t$ measures the time recorded on clocks moving on radial geodesics, i.e. $t$ measures proper time, $t=\tau$, and thus on radial geodesics we have $d s^{2}=-d t^{2}$. The sign given by $\varsigma$ indicates whether the geodesic clock is moving with increasing $\mathrm{R}(\varsigma=+1)$ or with decreasing $\mathrm{R}(\varsigma=-1)$. So that for an expanding Universe we have $\varsigma=+1$. Now we can make a coordinate transformation from $(T, R)$ to $(t, R)$ coordinates. In $(t, R)$ coordinates the radial geodesics are described by the 4 -velocity

$$
V^{\mu}(t, R)=\left(1, \varsigma\left(\mathcal{E}^{2}-A\right)^{\frac{1}{2}}, 0,0\right) .
$$

The resulting form of the line element is:

$$
\begin{aligned}
d s^{2} & =\mathcal{E}^{-2}\left(-A d t^{2}+d R^{2}-2 \varsigma\left(\mathcal{E}^{2}-A\right)^{\frac{1}{2}} d R d t\right)+R^{2} d \Omega^{2} \\
& =-d t^{2}+\mathcal{E}^{-2}\left(d R-\varsigma\left(\mathcal{E}^{2}-A\right)^{\frac{1}{2}} d t\right)^{2}+R^{2} d \Omega^{2} .
\end{aligned}
$$

Because $t$ measures time on clocks moving relative to $R=$ constant the metric is non-diagonal. The cosmic particles, e.g. the galaxies are assumed to follow radial geodesics. Without the embedded Schwarzschild mass, i.e. $m=0$, we require the Universe to be isotropic and homogeneous. The choice of coordinates ensures the isotropic condition. For a flat Universe model the homogeneity condition settles $\mathcal{E}$ [17]. First we note that for $m=0$ the coordinates are required to reduce to the Minkowskian form at $R=0$, i.e. $A=f=1$. Then from (3) we find that at $R=0$ the energy parameter $\mathcal{E}$ is given by the Lorentz factor $\gamma: \mathcal{E}=\left(1-v_{0}^{2}\right)^{-\frac{1}{2}}$, where $v_{0}$ is the coordinate velocity, $\frac{d R}{d T}$, of the reference particles at $R=0$; and because the space-time metric here is flat this is the velocity measured by an observer at $R=0$. Hence, $\mathcal{E}$ gives the energy to rest mass ratio for the cosmic particles. Particles with $\mathcal{E}<1$ will never reach $R=0$, thus making a hole in the cosmic fluid. While for $\mathcal{E}>1$ they will have some velocity, $v_{0}^{2}>0$, at $R=0$. This makes $R=0$ a source $(\varsigma=+1)$ or a sink $(\varsigma=-1)$ for cosmic particles. For a homogeneous Universe $R=0$ cannot have any special significance. Hence, we are lead to

$$
\mathcal{E}=1
$$

for a spatially flat Universe. If we embed a Schwarzschild mass into this model we get an inhomogeneous model, but we still have $\mathcal{E}=1$ [17. Gautreau argues 
that for a vacuum Universe, i.e. the de Sitter model to have a physical reference system we cannot set $\mathcal{E}=1$. The vacuum Universe in the non-diagonal metric (17) is discussed in 22. For a thorough investigation of the kinematics of the de-Sitter Universe see 23.

For a flat Universe model, i.e. $\mathcal{E}=1$, the surface of simultaneity $d t=0$ in (17) gives the flat metric.

$$
d l^{2}=d R^{2}+R^{2} d \Omega^{2} .
$$

In this case $R$ measures the actual distance between the cosmic particles.

We shall now consider $\mathcal{E}$ for open and closed models. To get a feeling for the coordinates in (7) we relate them to the commonly used comoving coordinates in cosmology. In these coordinates the metric has the form

$$
d s^{2}=-d t^{2}+a(t)^{2}\left(\frac{d r^{2}}{1-k r^{2}}+r^{2} d \Omega^{2}\right)
$$

where $a(t)$ is the expansion factor and the radial coordinate $r$ is constant along the trajectory of the cosmic particles, e.g. galaxies, and $t$ is the time measured on clocks moving with them. The sign $k$ gives the spatial topology (i.e. the global structure of the $t=$ constant surface): $k=+1$ leads to a spherical (closed) geometry, $k=-1$ gives a hyperbolic (open) geometry and $k=0$ describes an Euclidean (flat) geometry.

The time coordinates in (7) and (10) measure time on clocks moving with the cosmic particles. Thus we identify the time coordinates in (7) and (10). The radial coordinates are related by $R=a r$ and the energy parameter, $\mathcal{E}$, for the reference particles is given by $\mathcal{E}^{2}=1-k r^{2}=1-k C(t, R)$, where $C(t, R)$ is a function that is constant along the radial geodesics, i.e. $\mathcal{E}$ must be constant along the geodesics. The Einstein equations give this function up to a constant factor. The Hubble factor, $H \equiv \frac{1}{a} \frac{d a}{d t}$, is now given by

$$
\frac{d R}{d t}=H R
$$

\subsection{The Einstein equations for curvature coordinates}

The Einstein equations with cosmological constant $G^{\mu}{ }_{\nu}+\Lambda \delta^{\mu}{ }_{\nu}=8 \pi T^{\mu}{ }_{\nu}$ which we will need to deal with are:

$$
\begin{aligned}
& \frac{\partial(R(1-A))}{\partial R}=-8 \pi R^{2} T^{t}+\Lambda R^{2} \\
& \frac{\partial(R(1-A))}{\partial t}=8 \pi R^{2} T_{t}^{R} \\
& \frac{\partial(R(1-A))}{\partial R}+\varsigma\left(\mathcal{E}^{2}-A\right)^{-\frac{1}{2}} \frac{\partial(R(1-A))}{\partial t}+ \\
& 2 \varsigma\left(\frac{R A}{\mathcal{E}}\right)\left(\mathcal{E}^{2}-A\right)^{-\frac{1}{2}}\left(\frac{\partial \mathcal{E}}{\partial t}+\varsigma\left(\mathcal{E}^{2}-A\right)^{\frac{1}{2}} \frac{\partial \mathcal{E}}{\partial R}\right)=-8 \pi R^{2} T_{R}^{R}+\Lambda R^{2}
\end{aligned}
$$

These equations are valid in coordinates $x^{\mu}=(t, R, \theta, \phi)$ for an arbitrary energymomentum tensor $T_{\nu}^{\mu}$. The field equations involving second-order derivatives 


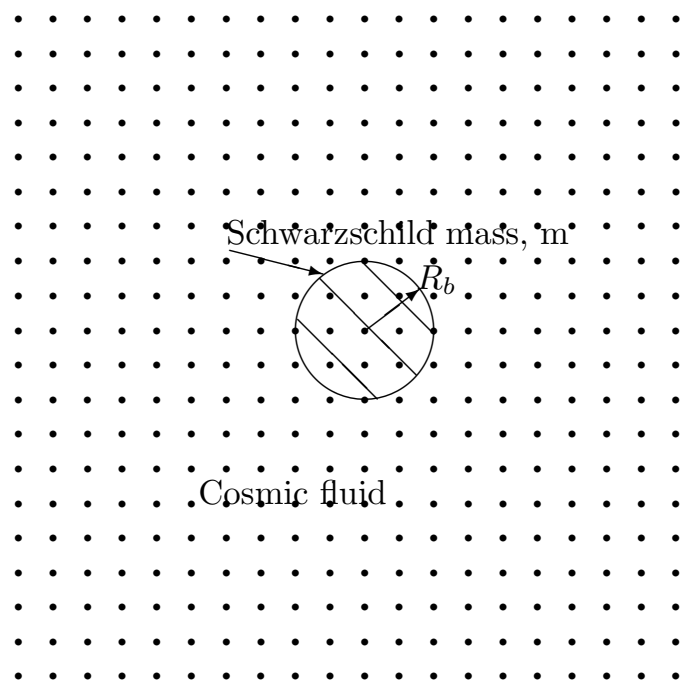

Figure 1: Schwarzschild mass embedded in a cosmic fluid.

are the angular part, for which $T_{\theta}^{\theta}=T_{\phi}^{\phi}$; these equations are contained in the divergence of the Einstein tensor, $G_{\nu ; \mu}^{\mu}=0$.

To proceed an energy-momentum tensor for the Universe with an embedded Schwarzschild mass is assumed. One may imagine the galaxies to be particles of a fluid and that this cosmic fluid fills the whole spacetime. ${ }^{3}$ The embedded Schwarzschild mass, $\mathrm{m}$, is placed with its center at $R=0$, and with boundary at $R=R_{b}$, see figure (11). As mentioned above the particles of the cosmic fluid are assumed to follow the radial geodesics $V^{\mu}$. The cosmic fluid is described as an ideal fluid. Thus, outside $R_{b}$ the energy-momentum tensor is:

$$
T^{\mu}{ }_{\nu}=(\rho+p) V^{\mu} V_{\nu}+p \delta^{\mu}{ }_{\nu}, \quad R>R_{b},
$$

where $\rho$ is the mass density and $p$ the pressure of the cosmic fluid. Inserting $V^{\mu}$ given in (6) into the energy-momentum tensor (15) gives the components:

$$
\begin{aligned}
& T_{t}^{t}=-\rho, \quad T_{R}^{R}=T_{\theta}^{\theta}=T_{\phi}^{\phi}=p, \\
& T_{R}^{t}=0, \quad T_{t}^{R}=-\varsigma\left(\mathcal{E}^{2}-A\right)^{\frac{1}{2}}(\rho+p) .
\end{aligned}
$$

Inside $R_{b}$ we will assume that the $t t$ component of the energy-momentum tensor can be written as

$$
T_{t}^{t}=-\rho+\rho_{t}^{t}, \quad R \leq R_{b},
$$

\footnotetext{
${ }^{3}$ The embedding of a Schwarzschild mass into a cosmological model has also been considered by Einstein and Straus 24 (the Swiss Cheese model). They looked at a cosmic dust fluid with a vacuum region $(\Lambda=0)$ with a Schwarzschild mass at the center. And showed that one can join the Schwarzschild metric smoothly onto the cosmic metric 10. An explicit form of this metric was found by Schücking [25] using "curvature" coordinates. For recent developments on this embedding see e.g. 19$] 26[27$.
} 
where $\rho$ is the energy density of the cosmic fluid and $\rho^{t}{ }_{t}$ is the part of the energymomentum tensor giving the energy density of the embedded Schwarzschild mass. If we assume that $\rho^{t}{ }_{t}$ is time independent this is the only component of the energy momentum tensor inside $R_{b}$ we will need. The constant embedded Schwarzschild mass, $\mathrm{m}$, bounded by $R_{b}$ is defined by:

$$
m=-4 \pi \int_{0}^{R_{b}} \rho^{t}{ }_{t} R^{2} d R .
$$

We note that setting $m=0$ leads to a description of cosmology in "curvature" coordinates. Let us also define a mass function $M(t, R)$ for the Universe:

$$
M(t, R)=4 \pi \int_{0}^{R} \rho(t, R) R^{2} d R
$$

where the integration is over a $t=$ constant surface. In terms of $M$ the density is given by

$$
\frac{\partial M(t, R)}{\partial R}=4 \pi R^{2} \rho(t, R)
$$

where $4 \pi R^{2}$ gives the area of a sphere centered on $R=0$.

In the case where we have an embedded mass $m$ the density $\rho$ of the cosmic fluid will have some $R$ dependence and we cannot bring $\rho$ outside the integrals. But for $m=0$ the cosmic fluid is homogeneous, and $\rho$ is only a function of $t$ and thus can be brought outside the integrals.

We now move on to solve the Einstein equations (12 14) outside $R_{b}$, with an energy-momentum tensor given by (16) and (17). We start by integrating (12) over a $t=$ constant surface. Using (18) and (19) this gives the metric function in the form

$$
A=1-\frac{2(m+M)}{R}-\frac{\Lambda}{3} R^{2},
$$

with the metric given in (7). Note that in general $M$ is a function of $t$ and $\mathrm{R}$.

We need to determine M. Inserting (21) into (13) gives

$$
\begin{aligned}
T_{t}^{R} & =\frac{1}{4 \pi R^{2}} \frac{\partial M}{\partial t} \\
& =\frac{1}{R^{2}} \int_{0}^{R} \frac{\partial \rho}{\partial t} R^{2} d R
\end{aligned}
$$

which gives the energy (mass) flux across a $R=$ constant surface. The $T_{t}^{R}$ component of the energy momentum tensor for an ideal fluid is given in (16). Equating these two expressions for $T_{t}^{R}$ and using (20) we find

$$
\frac{\partial M}{\partial t}+\varsigma\left(\mathcal{E}^{2}-A\right)^{\frac{1}{2}} \frac{\partial M}{\partial R}=-\varsigma 4 \pi R^{2} p\left(\mathcal{E}^{2}-A\right)^{\frac{1}{2}},
$$

which is the partial differential equation determining $M$. From (23) and (20), assuming $p \geq-\rho$, we see that for an expanding Universe $(\varsigma=+1)$ we have $\frac{\partial M}{\partial t} \leq 0$; and for a contracting Universe $(\varsigma=-1)$ we get $\frac{\partial M}{\partial t} \geq 0$. 
To proceed from here we assume an equation of state $p=p(\rho)$ for the cosmic fluid. We assume that the cosmic fluid obeys the barotropic equation of state

$$
p=\omega \rho, \quad \omega=\omega(t, R),
$$

e.g. $\omega=0$ gives a dust Universe, $\omega=\frac{1}{3}$ describes a radiation dominated Universe and $\omega=-1$ represents a vacuum Universe. Using (20) we get

$$
\frac{\partial M}{\partial t}+\varsigma(1+\omega)\left(\mathcal{E}^{2}-A\right)^{\frac{1}{2}} \frac{\partial M}{\partial R}=0 .
$$

Writing (23) in terms of $\rho$ [17] for a general Universe leads to

$$
R^{\frac{1}{2}} \int_{0}^{R} \frac{\partial \rho}{\partial \tau} R^{2} d R+\varsigma R^{2}(\rho+p)\left(\mathcal{E}^{2}-1+8 \pi \int_{0}^{R} \rho R^{2} d R+2 m+\frac{\Lambda}{3} R^{3}\right)^{\frac{1}{2}}=0
$$

Evaluating (23) along the geodesics we find

$$
\frac{d M}{d t}=-4 \pi R^{2} p \frac{d R}{d t}
$$

For dust, $p=0$, this gives $\frac{d M}{d t}=0$. So that $\mathrm{M}$ is constant along the geodesics.

Consider the vacuum case. For Lorentz invariant vacuum energy the energy momentum tensor is proportional to the metric [28, i.e. $T_{\mu \nu}=\rho_{\Lambda} g_{\mu \nu}$ with the vacuum energy density $\rho_{\Lambda}$ constant. Thus, this type of vacuum energy can be incorporated into the cosmological constant $\Lambda$; we have $\Lambda=8 \pi \rho_{\Lambda}$. So that here the embedding of a Schwarszchild mass into the de Sitter Universe is given by $M=0$. The trivial solution of (25) is $M=$ constant, and if this is a global solution then from the definition of $M$ in (19) and (20) this constant must be zero. Let now $\rho_{\Lambda}$ be part of the energy momentum tensor, i.e. included in M. For vacuum, $\omega=-1$, the second term in (25) or (26) vanish. This gives $\frac{\partial M}{\partial t}=0$ showing that $\mathrm{M}$ is time independent. For a homogeneous space $\rho_{\Lambda}$ must then be constant. Let us also briefly look at the Einstein equations using the diagonal metric (11). The above equations become for an arbitrary $T_{\nu}^{\mu}$ :

$$
\begin{aligned}
& A(R, T)=1+\frac{8 \pi}{R} \int_{0}^{R} T_{T}^{T} R^{2} d R-\frac{\Lambda}{3} R^{2}, \\
& T_{T}^{R}=-\frac{1}{R^{2}} \int_{0}^{R} \frac{\partial T_{T}^{T}}{\partial T} R^{2} d R, \\
& \ln f^{2}=8 \pi \int_{0}^{R} \frac{R}{A}\left(T_{R}^{R}-T_{T}^{T}\right) d R .
\end{aligned}
$$

The integration is now over a $T=$ constant surface. The coordinate transformation between the coordinate systems is given by (40) and (5). From (30) we see that all vacuum space-times have $f=1$. And for a Schwarzschild mass embedded in a de-Sitter Universe the metric (1) is given by $A=1-\frac{2 m}{R}-\frac{\Lambda}{3} R^{2}$.

Let us now consider $\mathcal{E}$. From the Einstein equation (14) and demanding that (23) is satisfied we find

$$
\frac{\partial \mathcal{E}}{\partial t}+\varsigma\left(\mathcal{E}^{2}-A\right)^{\frac{1}{2}} \frac{\partial \mathcal{E}}{\partial R}=0
$$


For geodesics this gives $\frac{d \mathcal{E}}{d t}=0$, which shows that the Einstein equations require that $\mathcal{E}$ is a constant along the streamlines of the cosmic fluid outside $R_{b}$. For a flat Universe model, with and without the embedded Schwarzschild mass, this constant must be set to $\mathcal{E}=1$. For a Universe with a non zero spatial curvature we see from eq. (25) that $\mathcal{E}$ can be written in terms of $M$ for a dust Universe. To recover standard cosmology we require that $\mathcal{E}^{2}-1$ is proportional to $M$. Hence, we can write

$$
\mathcal{E}^{2}=1-k \frac{2 M}{R_{i}},
$$

where $R_{i}=$ constant . This constant depends on which galaxy we wish to follow and thus it determines M. E.g. for a closed $(k=+1)$ Universe $R_{i}$ represents the maximum radius reached by a given galaxy. For further discussion of this and the open case see 29.

Next we will consider the velocity and the acceleration of the cosmic particles. From the geodesics in (6) the coordinate velocity is in general given by

$$
\frac{d R}{d t}=\left(\mathcal{E}^{2}-A\right)^{\frac{1}{2}}
$$

with the Hubble parameter given by $H=\frac{1}{R} \frac{d R}{d t}$. Inserting for A and $\mathcal{E}$ gives

$$
\left(\frac{d R}{d t}\right)^{2}=-k \frac{2 M}{R_{i}}+\frac{2(m+M)}{R}+\frac{\Lambda}{3} R^{2}
$$

where $M$ is constant for a dust $(p=0)$ Universe. Differentiating (34) and inserting (27) with $p=0$ we get the acceleration for a dust Universe,

$$
\frac{d^{2} R}{d t^{2}}=-\frac{m+M}{R^{2}}+\frac{\Lambda}{3} R .
$$

For $m=0$ we have a homogeneous cosmic fluid, thus we can find $M$ from considering the geodesics (34) and (35). If $m \neq 0$ the cosmic fluid is inhomogeneous and we must find $M$ by treating the coordinates, $t$ and $R$, as independent variables, i.e. from eq. (23). Also, we note that for $m=0$ equations (34) and (35) are equivalent to the Friedmann equations, i.e. the dynamical equations obtained from Einsten's field equations when using the comoving coordinates in (10). For $\Lambda=0$ (35) is identical to Newton's gravitational law, see [29].

For light the trajectories are given by $d s^{2}=0$, thus the paths of radially moving light are described by

$$
\frac{d R}{d t}=\left(\mathcal{E}^{2}-A\right)^{\frac{1}{2}} \pm \mathcal{E} .
$$

For a closed Universe model, $k=+1$, in the limit $R_{i} \rightarrow 2 M$ we have $\mathcal{E}^{2} \rightarrow 0$ giving a singularity in the metric (17). In this case (34) approaches (36), i.e. the galaxies approach the speed of light. This is the maximum size of the Universe 29. 


\section{Solutions}

\section{$3.1 m=0$}

For a general Universe without the embedded Schwarzschild mass we can bring $\rho$ outside the integrals above. The mass function, $M$, for the Universe defined in (19) becomes

$$
M=\frac{4 \pi}{3} R^{3} \rho(t) .
$$

For a dust Universe this gives the mass of the Universe inside $\mathrm{R}$ at time $t$. In the following we shall consider a flat Universe model, $\mathcal{E}=1$, such that $\mathrm{R}$ measures the proper distance between the cosmic particles. Eq. (26) then reduces to:

$$
\frac{d \rho}{d t}=-\varsigma(\rho+p)(24 \pi \rho+3 \Lambda)^{\frac{1}{2}}
$$

Along the geodesics (see (27)) eq. (38) reads

$$
\frac{d \rho}{d t}=-3(\rho+p) \frac{1}{R} \frac{d R}{d t},
$$

independent of $\Lambda$ (and $\mathcal{E}$ ). From (38) and (39) we find a 1-parameter family of solutions. We assume an equation of state of the form $p=\omega \rho$ with $\omega=$ constant. Integration of (39) then gives $\rho(t)$ in terms of the geodesics, $R(t)$ :

$$
\rho(t)=b R(t)^{-3(1+\omega)} \text {. }
$$

This expression for the density is in fact valid for all the FLRW Universe models, i.e. regardless of $\Lambda$ and $\mathcal{E}$. For dust $\omega=0$ which inserted into (40) gives $\rho \propto R^{-3}$, i.e. $\mathrm{M}$ is constant, for radiation we have $\omega=\frac{1}{3}$ and we get $\rho \propto R^{-4}$. For vacuum, $\omega=-1$, we get $\rho=$ constant which is also obtained from eq. (38); the expansion of the vacuum Universe is found from eq. (34):

$$
\begin{array}{ll}
R \propto \cosh \sqrt{\frac{\Lambda}{3}} t, & \mathcal{E}<1, \\
R \propto e^{\sqrt{\frac{\Lambda}{3}} t}, & \mathcal{E}=1, \\
R \propto \sinh \sqrt{\frac{\Lambda}{3}} t, & \mathcal{E}>1 .
\end{array}
$$

In the following we assume that $\omega \neq-1$. We note that the density $\rho$ does not depend on the integration constant $b$. This constant depends on which geodesic (galaxy) we wish to follow, (see below). Thus, we can relate $b$ to the comoving coordinate $r$ in (10). Normalizing the expansion factor, $a$, so that $a_{0}=1$, where the index 0 refers to the present time, we get $b=r^{3(1+\omega)} \rho_{0}$. For a dust Universe $\mathrm{M}$ is constant and we find that $M=\frac{4 \pi}{3} b$. That is, $b$ is a measure of the mass inside a comoving radius $r=$ constant. Alternatively, one can regard $b$ as a measure of the energy of a cosmic particle relative to $R=0, t=0$.

Solving eq. (38) for $\Lambda=0$ gives

$$
\rho=\frac{1}{(1+\omega)^{2} 6 \pi t^{2}} .
$$




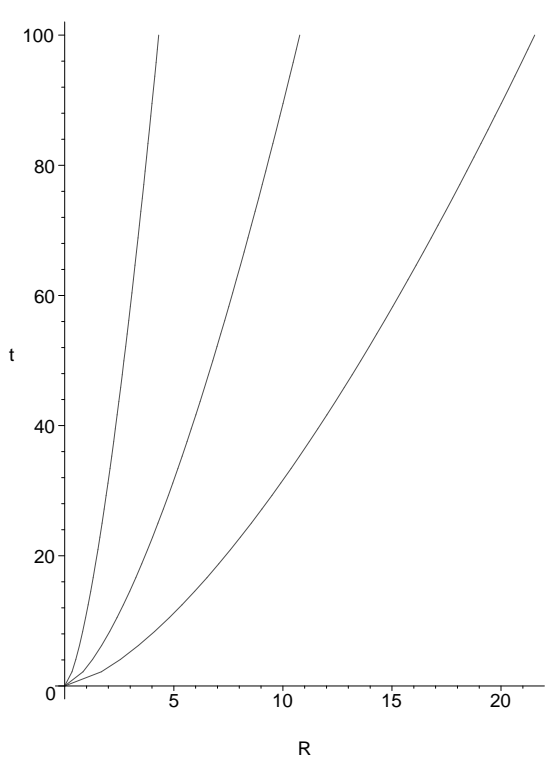

(a) Einstein-de Sitter

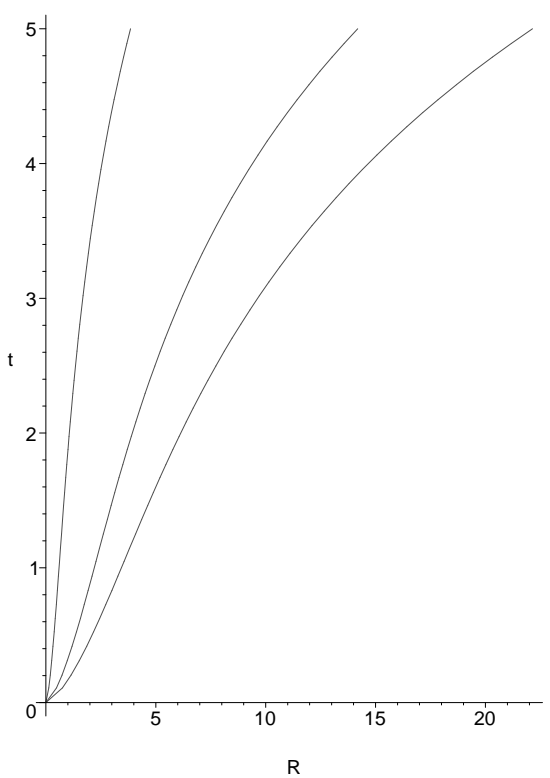

(b) Friedmann-Lemaître

Figure 2: Graphs showing the radial timelike geodesics for different values of $b$ in a Universe containing (a) dust, and (b) dust and vacuum energy.

We have set the integration constant to zero so that the big bang is placed at $R(t=0)=0$. Eq. (44) shows that the density of all ideal fluids, in a flat Universe, with $p=\omega \rho$ are proportional to $t^{-2}$. Combining (40) and (44) we find the geodesics:

$$
R=\left(b(1+\omega)^{2} 6 \pi t^{2}\right)^{\frac{1}{3(1+\omega)}} .
$$

The geodesics for for the Einstein-de Sitter model, $\omega=0$, are displayed in fig[2] where the geodesic $R=0$ represents the trajectory of e.g. our galaxy. Inserting (44) and (37) into (21) leads to the metric:

$$
A=1-\left(\frac{2 R}{3(1+\omega) t}\right)^{2}
$$

and the Hubble parameter

$$
H=(1-A)^{\frac{1}{2}} \frac{1}{R}=\frac{2}{3(1+\omega) t},
$$

where the line-element is given in (7).

The current standard model of the Universe is the flat Friedmann-Lemaitre model, which is a Universe model with dust and Lorentz invariant vacuum energy; i.e. $\omega=0$ and $\Lambda>0$. A pedagogical presentation of this model is given in 30 . 
For a non zero $\Lambda$ there are two cases: $\Lambda>0$ and $\Lambda<0$. Integrating (38) for $\Lambda>0$ we get

$$
\rho=\frac{\Lambda}{4 \pi \cosh ((1+\omega) \sqrt{3 \Lambda} t)-4 \pi} .
$$

Taking the series for cosh we get (44) in the limit $\Lambda \rightarrow 0$. Eq. (48) can also be written

$$
\rho=\frac{\Lambda}{8 \pi \sinh ^{2}\left((1+\omega) \frac{\sqrt{3 \Lambda}}{2} t\right)} .
$$

Thus, from eq. (21) the metric (7) is given by

$$
A=1-\frac{\Lambda}{3} R^{2} \operatorname{coth}^{2}\left((1+\omega) \frac{\sqrt{3 \Lambda}}{2} t\right)
$$

and the Hubble parameter

$$
H=\sqrt{\frac{\Lambda}{3}} \operatorname{coth}\left((1+\omega) \frac{\sqrt{3 \Lambda}}{2} t\right) .
$$

From (40) the trajectories are

$$
\begin{aligned}
R & =\left(b \frac{4 \pi \cosh ((1+\omega) \sqrt{3 \Lambda} t)-4 \pi}{\Lambda}\right)^{\frac{1}{3(1+\omega)}} \\
& =\left(b \frac{8 \pi \sinh ^{2}\left((1+\omega) \frac{\sqrt{3 \Lambda}}{2} t\right)}{\Lambda}\right)^{\frac{1}{3(1+\omega)}}
\end{aligned}
$$

which are shown for the Friedmann-Lemaitre model, i.e. $\omega=0$, in fig 2 Eq. (35) then implies that the expansion of the Universe becomes accelerated for

$$
\Lambda>4 \pi \rho
$$

or in terms of the Lorentz invariant vacuum energy density: $\rho_{\Lambda}>\frac{1}{2} \rho, 30$.

Solving (38) for $\Lambda<0$ we obtain the solution

$$
\rho=\frac{-3 \Lambda}{24 \pi \cos ^{2}\left(-\frac{\sqrt{-3 \Lambda}}{2}(1+\omega) t\right)},
$$

where from (40) $\rho \propto R^{-3(1+\omega)}$. This is an oscillating Universe with singularities at $R=0, t=\frac{2 \pi n}{\sqrt{-3 \Lambda}(1+\omega)} ; \mathrm{n}$ is a half-integer.

The partial differential equation (25) for $M$ must also give these solutions. We now consider this equation for $\Lambda=0$. Let us set $Y=2 m+2 M$. In terms of $Y$ equation (25) for an expanding Universe becomes

$$
R^{\frac{1}{2}} \frac{\partial Y}{\partial \tau}+(1+\omega) Y^{\frac{1}{2}} \frac{\partial Y}{\partial R}=0
$$


which is easily solved by separation of variables. The solution is

$$
A=1-\frac{2(m+M)}{R}=1-\frac{\left(\frac{2 c R^{\frac{3}{2}}+3 c_{2}}{3(1+\omega)\left(c t+c_{1}\right)}\right)^{2}}{R},
$$

where $c$ is the separation constant and $c_{1}$ and $c_{2}$ are integration constants. In the limit $R \rightarrow \infty$ the solution should give eq. (46), this requires that $c_{1}=0$. Consider now the case where $\mathrm{R}$ is finite and $t \rightarrow \infty$. We would in this case, for an embedded mass, expect the solution to approach the Schwarzschild solution. But here the solution goes towards Minkowski spacetime, indicating that our solution represents a pure $(m=0)$ Universe. Thus, we should set $c_{2}=0$; then the separation constant cancels and the solution reduces to (46). This method of solving (25) cancels out the effect of $\mathrm{m}$. But from this we see that for $m=0$ equation (25) gives the required solution.

\section{$3.2 m \neq 0$}

We now turn to the case where we have an embedded mass, $m \neq 0$. In 17 no discussion was made on solutions of equation (25). We consider it for a flat Universe with $\Lambda=0$.

To find solutions that include the effect of the embedded mass $m$ we shall look at approximations. We will find solutions valid close to $\mathrm{m}$ and at large distances from $\mathrm{m}$.

We expand the square root

$$
(2 m+2 M)^{\frac{1}{2}}=(2 m)^{\frac{1}{2}}\left(1+\frac{1}{2} \frac{M}{m}-\frac{1}{2 \cdot 4}\left(\frac{M}{m}\right)^{2}+\ldots\right),
$$

thus defining the function $F$ :

$$
(2 m+2 M)^{\frac{1}{2}}=(2 m)^{\frac{1}{2}}+2 F .
$$

From (25) we get the following equation for $F$ :

$$
R^{\frac{1}{2}} \frac{\partial F}{\partial \tau}+(1+\omega)(2 m)^{\frac{1}{2}} \frac{\partial F}{\partial R}+(1+\omega) 2 F \frac{\partial F}{\partial R}=0,
$$

which has two approximations:

$$
\begin{array}{ll}
R^{\frac{1}{2}} \frac{\partial F}{\partial \tau}+(1+\omega)(2 m)^{\frac{1}{2}} \frac{\partial F}{\partial R} \approx 0, & 2 F \ll(2 m)^{\frac{1}{2}}, \\
R^{\frac{1}{2}} \frac{\partial F}{\partial \tau}+(1+\omega) 2 F \frac{\partial F}{\partial R} \approx 0, & 2 F \gg(2 m)^{\frac{1}{2}} .
\end{array}
$$

Here (61) gives an approximate solution for the spacetime close to the embedded mass $\mathrm{m}$, and (62) gives an approximate solution for the spacetime far from $m$. Both equations are easily solved by separation of variables.

From equation (62) we find the following solution:

$$
(2 m+2 M)^{\frac{1}{2}}=(2 m)^{\frac{1}{2}}+\frac{2 R^{\frac{3}{2}}}{3(1+\omega) t},
$$


where we have set the integration constants to zero. The metric is given by

$$
A=1-\left(\left(\frac{2 m}{R}\right)^{\frac{1}{2}}+\frac{2 R}{3(1+\omega) t}\right)^{2} .
$$

This solution is valid for $2 F \gg(2 m)^{\frac{1}{2}}$ which is

$$
\frac{2 R^{3}}{9(1+\omega)^{2} t^{2}} \gg m
$$

i.e. where the Shcwarzschild field is much weaker than the background (see (37) and (44)). Taking the limit $R \rightarrow \infty$ this solution approaches the solution (46) obtained for $m=0$. Since the solution is only valid far from the embedded mass, $\mathrm{M}$ is now not a global quantity; i.e. it does not represent the mass of the Universe. From (20) we find the density $\rho$ of the cosmic fluid with an embedded mass,

$$
\rho(t, R)=\frac{1}{(1+\omega)^{2} 6 \pi t^{2}}+\frac{(2 m)^{\frac{1}{2}}}{(1+\omega) 4 \pi R^{2} t} .
$$

The last term gives the deviation from the density (44) of the cosmic fluid in a FRW Universe. We get the geodesics by inserting (63) into (34). Thus, far from $m$ we have

$$
H R=\frac{d R}{d t}=\left(\frac{2 m}{R}\right)^{\frac{1}{2}}+\frac{2 R}{3(1+\omega) t} .
$$

The solution for $\omega=0$ is

$$
R=\left(\frac{3}{2} \sqrt{2 m} \ln t+c\right)^{\frac{2}{3}} t^{\frac{2}{3}}
$$

where $c$ is the integration constant. We note that for $t \rightarrow 0$ we get $R \rightarrow 0$. Using eq. (65) we see that this solution is valid for:

$$
t \gg e^{p_{1}}, \quad p_{1}=1-\frac{2 c}{3(2 m)^{\frac{1}{2}}}
$$

and

$$
t \ll e^{p_{2}}, \quad p_{2}=-1-\frac{2 c}{3(2 m)^{\frac{1}{2}}} .
$$

For $\omega \neq 0$ the solution is

$$
R=\left(\frac{3}{2} \sqrt{2 m}\left(\frac{1}{\omega}+1\right) t+c t^{\frac{1}{1+\omega}}\right)^{\frac{2}{3}} .
$$

Valid for

$$
t \ll\left(\frac{2 c}{3(2 m)^{\frac{1}{2}}\left(\frac{\omega^{2}-\omega}{1+\omega}\right)}\right)^{\frac{1+\omega}{\omega}}
$$


For $m=0$ the solutions (68) and (71) reduces to eq. (45) with the identification $c=((1+\omega) \sqrt{6 \pi b})^{\frac{1}{\omega+1}}$.

Next consider the approximation (61). This equation has solution

$$
(2 m+2 M)^{\frac{1}{2}}=(2 m)^{\frac{1}{2}}+a e^{b x},
$$

where

$$
x=\frac{2 R^{\frac{3}{2}}}{3(1+\omega)(2 m)^{\frac{1}{2}}}-t,
$$

$a$ is an integration constant and $b$ is the separation constant. This solution is valid for

$$
b x \ll \ln \frac{(2 m)^{\frac{1}{2}}}{a} .
$$

The metric function A becomes

$$
A=1-\frac{\left((2 m)^{\frac{1}{2}}+a e^{b x}\right)^{2}}{R} .
$$

For a finite $\mathrm{R}$ in the limit $t \rightarrow \infty$ we get $A \rightarrow 1-\frac{2 m}{R}$, i.e. the solution approaches the Schwarzschild solution. Also, for $a=0$, which gives $M=0$, the second term is zero and we are left with the Schwarzschild field. Considering (73) to first order in $\frac{M}{m}$ this solution reduces to

$$
M=(2 m)^{\frac{1}{2}} a e^{b x} .
$$

If we assume $M \ll m$ in (25) and ignore the non-linear term completely we can write $R^{\frac{1}{2}} \frac{\partial M}{\partial t}+(2 m)^{\frac{1}{2}} \frac{\partial M}{\partial R}=0$ which has solution (77). From (73) and (34) we have the geodesics close to $m$ approximated by

$$
H R=\frac{d R}{d t}=\left(\frac{2 m}{R}\right)^{\frac{1}{2}}+\frac{a e^{b x}}{R^{\frac{1}{2}}} .
$$

If we write this in terms of $x$ and then insert $Z=\frac{2 F}{a}=e^{b x}$, eq. (178) can be written

$$
\frac{d Z}{d t}=\frac{a b Z^{2}-b \omega(2 m)^{\frac{1}{2}} Z}{(1+\omega)(2 m)^{\frac{1}{2}}},
$$

which is easily integrated to find the trajectories. For dust, $\omega=0$, the geodesics are

$$
R(t)=\left(\frac{3(2 m)^{\frac{1}{2}}}{2} t-\frac{3(2 m)^{\frac{1}{2}}}{2 b} \ln \left(c_{1}-\frac{a b}{(2 m)^{\frac{1}{2}}} t\right)\right)^{\frac{2}{3}}
$$

where $c_{1}$ is the integration constant. If we demand that $R(t=0)=0$ we get $c_{1}=1$. From eq. (75) we find that this solution is valid for

$$
t \ll \frac{1}{b}\left(\frac{(2 m)^{\frac{1}{2}} c_{1}}{a}-1\right) .
$$

Thus, for this to be valid in an expanding Universe we must have $\sqrt{2 m} \gg a$. 
For $\omega \neq 0$ the solution is

$$
R(t)=\left(\frac{3(2 m)^{\frac{1}{2}}}{2} t+\frac{3(1+\omega)(2 m)^{\frac{1}{2}}}{2 b} \ln \left(\frac{\omega(2 m)^{\frac{1}{2}}}{a c_{2}+a e^{-\frac{\omega}{1+\omega} b t}}\right)\right)^{\frac{2}{3}} .
$$

Putting $R(t=0)=0$ gives the integration constant $c_{2}=\frac{\omega(2 m)^{\frac{1}{2}}}{a}-1$. This solution is valid for

$$
e^{\frac{\omega}{1+\omega} b t} \gg \frac{\omega-1}{c_{2}}
$$

By setting $a=0$ in (80), or (82), we are left with the radial Schwarzschild geodesics.

\section{Singular shells in isotropic Universe models}

We are considering a Universe containing, in addition to the cosmic fluid and $\Lambda$, energy confined to a surface (or rather an hypersurface). That is, we are dealing with situations where we have a shell of energy where the thickness, $\vartheta$, of the shell can be ignored; i.e. mathematically we let the thickness go to zero, $\vartheta \rightarrow 0$. This is the thin shell approximation. The energy momentum tensor for such a spacetime can be split into three parts. A general $T_{\alpha \beta}$ can therefore be written

$$
T_{\alpha \beta}=S_{\alpha \beta} \delta(y)+T_{\alpha \beta}^{+} \theta(y)+T_{\alpha \beta}^{-} \theta(-y),
$$

here $y$ is an orthogonal coordinate such that $\frac{\partial}{\partial y}=\mathbf{n}$ is a normalized normal vector and $y=0$ at the hypersurface. $\delta(y)$ is a delta function and $\theta(y)$ is the step function. In the previous section we discussed the embedding of a Schwarzschild mass into a cosmological model. Now we wish to obtain the relativistic equation of motion for a thin shell in this ambient space-time. From (84) we see that the shell contributes with a delta function singularity to the energy momentum tensor and thus does not follow geodesics in the background spacetime. In the thin shell approximation the energy-momentum tensor $S_{\alpha \beta}$ of the surface is defined as the integral over the thickness of the surface when the thickness $\vartheta$ goes to zero

$$
S_{\alpha \beta}=\lim _{\vartheta \rightarrow 0} \int_{-\frac{\vartheta}{2}}^{\frac{\vartheta}{2}} T_{\alpha \beta} d y
$$

\subsection{Israel's Formalism: The metric junction method}

To deal with the situation described above we use Israel's formalism [1]. In this section we give a review.

The spacetime manifold $\mathcal{M}$ is split into two parts, $\mathcal{M}^{+}$and $\mathcal{M}^{-}$, by a hypersurface $\Sigma$. That is, $\mathcal{M}^{+} \cup \mathcal{M}^{-}=\mathcal{M}$ with a common boundary: $\partial \mathcal{M}^{+} \cap$ $\partial \mathcal{M}^{-}=\Sigma$. 
The energy-momentum content of spacetime is coupled to the geometry through Einstein's field equations. In the regions $\mathcal{M}^{+}$and $\mathcal{M}^{-}$outside the hypersurface $\Sigma$ we assume that

$$
G_{\mu \nu}^{ \pm}=8 \pi T_{\mu \nu}^{ \pm}
$$

where + and - means the tensor evaluated in $\mathcal{M}^{+}$and $\mathcal{M}^{-}$, respectively. Thus, if $T_{\mu \nu}^{ \pm}$is given, the metrics for the two regions outside $\Sigma$ are obtained by solving (866). The line elements of the two regions are written

$$
d s_{ \pm}^{2}=g_{\mu \nu}^{ \pm} d x_{ \pm}^{\mu} d x_{ \pm}^{\nu} .
$$

On the hypersurface $\Sigma$ we denote the intrinsic coordinates by $\xi^{j}$, and the intrinsic (induced) metric is

$$
d s_{\Sigma}^{2}=h_{i j} d \xi^{i} d \xi^{j} .
$$

We use Greek indices to run over the coordinates of $\mathcal{M}^{ \pm}$( $d$ dimensional) while Latin indices run over the intrinsic coordinates of $\Sigma(d-1$ dimensional).

The equations for the hypersurface are given by the embeddings $\phi^{ \pm}$:

$$
x_{ \pm}^{\mu}=\phi_{ \pm}^{\mu}\left(\xi^{i}\right)
$$

where $x_{ \pm}^{\mu}$ are $c^{r}$ functions with $r \geq 4{ }^{4}$ To glue together $\mathcal{M}^{+}$and $\mathcal{M}^{-}$along the common boundary $\Sigma$ we require that the two metrics, $g_{\mu \nu}^{+}$and $g_{\mu \nu}^{-}$, induce the same intrinsic metric on $\Sigma$ :

$$
h_{i j}=g_{\mu \nu}^{+} \frac{\partial x_{+}^{\mu}}{\partial \xi^{i}} \frac{\partial x_{+}^{\nu}}{\partial \xi^{j}}=g_{\mu \nu}^{-} \frac{\partial x_{-}^{\mu}}{\partial \xi^{i}} \frac{\partial x_{-}^{\nu}}{\partial \xi^{j}} .
$$

We note that the junction is independent of the embeddings $x_{ \pm}^{\mu}$, which do not need to join continuously at the hypersurface. This is an essential property of Israel's formalism: We are free to choose coordinates in $\mathcal{M}^{+}$and $\mathcal{M}^{-}$, independently.

Let $\mathbf{n}$ be the unit normal to the hypersurface $\Sigma$, which is defined to point from $\mathcal{M}^{-}$to $\mathcal{M}^{+}$, such that:

$$
\mathbf{n} \cdot \mathbf{n}=\left.g_{\mu \nu} n^{\mu} n^{\nu}\right|^{ \pm}=\epsilon=\left\{\begin{array}{l}
+1 \\
-1
\end{array}\right.
$$

$\epsilon=+1$ gives a spacelike $\mathbf{n}$ and thus a timelike hypersurface $\Sigma$. $\epsilon=-1$ gives a timelike $\mathbf{n}$ and thus a spacelike $\Sigma$. For $\epsilon=0$ we call the hypersurface a null surface $^{5}$. We shall only consider timelike surfaces, $\epsilon=+1$.

The induced metric on $\Sigma$ can also be written in terms of $x_{ \pm}^{\mu}$. We have $h_{ \pm}^{\mu \nu}=h^{i j} \frac{\partial x_{ \pm}^{\mu}}{\partial \xi^{i}} \frac{\partial x_{ \pm}^{\nu}}{\partial \xi^{j}}$. In terms of the normal vector the induced metric becomes

$$
h_{ \pm}^{\mu \nu}=g_{ \pm}^{\mu \nu}-\epsilon n_{ \pm}^{\mu} n_{ \pm}^{\nu}
$$

\footnotetext{
${ }^{4}$ The continuity equations involves the 4 th derivative of $x_{ \pm}^{\mu}$.

${ }^{5}$ The formalism given here breaks down for this case, for a treatment of null surfaces see 213 31.
} 
$h_{ \pm}^{\mu \nu}$ defines a projection operator, $h_{\alpha}^{\mu} h_{\nu}^{\alpha}=h_{\nu}^{\mu}$, that picks out the part of a tensor that lies in the tangent space of $\Sigma$.

The extrinsic curvature tensor, $\mathbf{K}$, is essential to the formalism. This tensor is a measure of how the hypersurface curves in the surrounding spacetime $\mathcal{M}^{+}$ and $\mathcal{M}^{-}$. It is defined as the covariant derivative of the normal vector $\mathbf{n}$ with respect to the connection in $\mathcal{M}^{+}$and $\mathcal{M}^{-}$along the direction of the tangent vectors on $\Sigma$. Since $\mathbf{n}$ is everywhere normal to $\Sigma$ and of constant magnitude, the variation of $\mathbf{n}$ and thus $\mathbf{K}$ will be entirely in the tangent space of $\Sigma$. Also, since there is a delta function singularity in the energy momentum tensor at $\Sigma$, we get a discontinuity in $\mathbf{K}$ at $\Sigma\left(\delta(y)=\theta^{\prime}(y)\right)$. The extrinsic curvature tensors $K_{i j}^{+}$and $K_{i j}^{-}$in $\mathcal{M}^{+}$and $\mathcal{M}^{-}$, respectively, are thus given by

$$
K_{i j}^{ \pm}=-\left.\frac{\partial x^{\alpha}}{\partial \xi^{i}} \mathbf{e}_{\alpha} \cdot \frac{\partial x^{\beta}}{\partial \xi^{j}} \nabla_{\beta} \mathbf{n}\right|^{ \pm} .
$$

Note the sign convention. We can express $K_{i j}$ in terms of the Christoffel symbols of $\mathcal{M}^{ \pm}$. Using $\nabla\left(\mathbf{n} \cdot \frac{\partial x^{\alpha}}{\partial \xi^{i}} \mathbf{e}_{\alpha}\right)=0$ we have

$$
K_{i j}^{ \pm}=\left.\mathbf{n} \cdot \frac{\partial x^{\alpha}}{\partial \xi^{i}} \nabla_{\alpha}\left(\frac{\partial x^{\beta}}{\partial \xi^{j}} \mathbf{e}_{\beta}\right)\right|^{ \pm}=\left.\left(\frac{\partial^{2} x^{\mu}}{\partial \xi^{i} \partial \xi^{j}}+\Gamma_{\alpha \beta}^{\mu} \frac{\partial x^{\alpha}}{\partial \xi^{i}} \frac{\partial x^{\beta}}{\partial \xi^{j}}\right) n_{\mu}\right|^{ \pm}
$$

We see that $K_{i j}$ is symmetric and represents 4 -scalars in $\mathcal{M}^{ \pm}$. In terms of the projection operator (92) we have $K_{\mu \nu}^{ \pm}=-\left.h_{\mu}^{\alpha} h_{\nu}^{\beta} n_{\alpha ; \beta}\right|^{ \pm}$and $K_{i j}^{ \pm}=\left.K_{\mu \nu} \frac{\partial x^{\mu}}{\partial \xi^{i}} \frac{\partial x^{\nu}}{\partial \xi^{j}}\right|^{ \pm}$.

In Gaussian normal coordinates the metric is given by

$$
d s^{2}=d n^{2}+h_{i j}\left(\xi^{i}, n\right) d \xi^{i} d \xi j,
$$

where $\Sigma$ is located at $n=0$ and the induced metric on $\Sigma$ is $h_{i j}\left(\xi^{i}, 0\right)=h_{i j}\left(\xi^{i}\right)$. In this case the extrinsic curvature tensor is simply given by $K_{i j}=\Gamma_{i j}^{n}=$ $-\frac{1}{2} h_{i j, n}$ and $K_{j}^{i}=-\Gamma_{n j}^{i}=-h^{i k} \frac{1}{2} h_{k j, n}$.

The Einstein equations on the hypersurface are:

$$
\begin{aligned}
-\frac{1}{2} \epsilon^{(3)} R+\left.\frac{1}{2}\left(K^{2}-K_{l m} K^{l m}\right)\right|^{ \pm} & =8 \pi T_{n n}^{ \pm} \\
-\left.\left(K_{i}{ }^{l}{ }_{\mid l}-K_{\mid i}\right)\right|^{ \pm} & =8 \pi T_{i n}^{ \pm} \\
{ }^{(3)} G_{i j}+\left.\epsilon\left(K_{i j}-g_{i j} K\right)_{, n}\right|^{ \pm}-\left.3 \epsilon K_{i j} K\right|^{ \pm} & \\
+\left.2 \epsilon K_{i}{ }^{l} K_{j l}\right|^{ \pm}+\left.\epsilon \frac{1}{2} g_{i j}\left(K^{2}+K_{l m} K^{l m}\right)\right|^{ \pm} & =8 \pi T_{i j}^{ \pm},
\end{aligned}
$$

Integrating (96) and (97) across the shell in the thin shell approximation we find $S_{i n}=S_{n n}=0$. That is, $\mathbf{S}$ has no normal components to the shell. We have $S_{\mu \nu}^{ \pm}=\left.h_{\mu}^{\alpha} h_{\nu}^{\beta} S_{\alpha \beta}\right|^{ \pm}$and

$$
S_{i j}=\left.\frac{\partial x_{ \pm}^{\alpha}}{\partial \xi^{i}} \frac{\partial x_{ \pm}^{\beta}}{\partial \xi^{j}} S_{\alpha \beta}\right|^{ \pm} .
$$

Integrating (98) we arrive at the equation of motion for the surface:

$$
\left[K_{i j}\right]-[K] g_{i j}=8 \pi S_{i j}
$$


By contraction

$$
\left[K_{i j}\right]=8 \pi\left(S_{i j}-\frac{1}{2} S g_{i j}\right)
$$

These equations are called the Lanczos equations and they say that the surface energy-momentum tensor is given by the difference in the embeddings of $\Sigma$ in $\mathcal{M}^{ \pm}$. The bracket operation [] gives the discontinuity of a tensor at $\Sigma$

$$
[T]=T^{+}-T^{-} .
$$

In the same way we define the average \{\} as

$$
\{T\}=\frac{1}{2}\left(T^{+}+T^{-}\right) .
$$

We also note two relations between these definitions:

$$
\begin{gathered}
{[T S]=[T]\{S\}+\{T\}[S]} \\
\{T S\}=\{T\}\{S\}+\frac{1}{4}[T][S] .
\end{gathered}
$$

Using [ ] on the Einstein equations (96) and (97) along with the Lanczos equation (100) and equation (104) we find:

$$
S_{l m}\left\{K^{l m}\right\}+\left[T_{n n}\right]=0 .
$$

The discontinuity $\left[T_{n n}\right]$ gives the pressure exerted normal to the surface by the bulk. Contracting eq. (101) with $S_{i j}$ we get

$$
S_{l m}\left[K^{l m}\right]=8 \pi\left(S_{i j} S^{i j}-\frac{1}{2} S^{2}\right) .
$$

The contraction $S_{l m} K_{ \pm}^{l m}$ gives the normal component of the divergence of $\mathbf{S}$ with respect to the connection on $\mathcal{M}^{ \pm}$.

$$
\left.S_{; \beta}^{\alpha \beta}\right|^{ \pm}=\left.\left(\frac{\partial \phi^{\alpha}}{\partial \xi^{i}} S^{i l}{ }_{\mid l}+S_{l m} K^{l m} n^{\alpha}\right)\right|^{ \pm},
$$

where $\mid$ denotes covariant derivative with respect to the intrinsic connection on $\Sigma$ (i.e. the metric connection defined by $h_{i j}$ ). The normal part is

$$
\left.n_{\alpha} S_{; \beta}^{\alpha \beta}\right|^{ \pm}=S_{l m} K_{ \pm}^{l m} .
$$

Combining (106) and (107) we can separate $S_{l m} K_{+}^{l m}$ and $S_{l m} K_{-}^{l m}$ 13]. In a spherically symmetric space-time with the surface consisting of an ideal fluid we may separate the time components of $K_{+}^{l m}$ and $K_{-}^{l m}$.

The continuity equation for the surface is

$$
S_{i}^{l}{ }^{l}{ }^{\prime}+\left[T_{i n}\right]=0
$$


Contracting $\left[T_{i n}\right]$ with $u^{i}$ we get the momentum-flux of the bulk as measured by a comoving observer to the surface. Contracting with a spacelike tangent vector, $x^{i}$, this term gives the tangential force, in the $x^{i}$ direction, exerted on the surface by the bulk.

Taking the covariant derivative of the 4-velocity $\mathbf{u}_{ \pm}$in the direction of $\mathbf{u}_{ \pm}$ with respect to the connection on $\mathcal{M}^{ \pm}$we obtain the 4 -acceleration $\mathbf{a}_{ \pm}$. As viewed from $\mathcal{M}^{ \pm}$

$$
\left.a^{\alpha} \vec{e}_{\alpha}\right|^{ \pm}=\left.\nabla_{\vec{u}} \vec{u}\right|^{ \pm}=u_{\mid m}^{l} u^{m} \vec{e}_{l}+\left.K_{l m} u^{l} u^{m} \vec{n}\right|^{ \pm} .
$$

The normal component of the 4 -acceleration of the shell as viewed from $\mathcal{M}^{ \pm}$is thus

$$
\left.a^{\alpha} n_{\alpha}\right|^{ \pm}=K_{l m}^{ \pm} u^{l} u^{m}
$$

which is in general non-zero.

Applying \{\} on (96) and (97) gives the two constraints:

$$
\begin{aligned}
& { }^{(3)} R-\left\{K^{2}\right\}+\left\{K_{l m}\right\}\left\{K^{l m}\right\}=-16 \pi^{2}\left(S_{l m} S^{l m}+S^{2}\right)-16 \pi\left\{T_{n n}\right\}(113 \\
& \left\{K_{i \mid l}^{l}\right\}-\left\{K_{, i}\right\}=-8 \pi\left\{T_{i n}\right\}
\end{aligned}
$$

\subsection{Spherically symmetric thin shells}

We will look at a spherically symmetric shell consisting of an ideal fluid. The spherical symmetry implies that we can write the line-element, using proper time on the shell, as:

$$
d s_{\Sigma}^{2}=-d \tau^{2}+R(\tau)^{2} d \Omega^{2}
$$

where $R(\tau)$ is the expansion factor for the hypersurface, and the proper area is given by

$$
A_{\Sigma}=4 \pi R^{2} .
$$

The energy-momentum tensor for the shell consisting of an ideal fluid is

$$
S_{i j}=(\sigma+p) u_{i} u_{j}+p g_{i j},
$$

where $\sigma$ is the mass (energy) density of the surface and $p$ is the tangential pressure of the surface. Since we use proper time the 4-velocity of a comoving observer is $u^{i}=(1,0,0)$. The mass, $\mu$, of the surface is

$$
\mu=4 \pi R^{2} \sigma
$$

The angular coordinates define tangent vectors to the surface. Thus the radial coordinates must join continuously on the hypersurface. Hence, the equation of the surface is given by

$$
R_{ \pm}=R(\tau)
$$

henceforth omitting the subscripts on $\mathrm{R}$. The line element in the spacetimes $\mathcal{M}^{+}$ and $\mathcal{M}^{-}$when including the effect of a Schwarzschild mass on the background is given in (7). That is, 


$$
\begin{aligned}
d s_{ \pm}^{2} & =\mathcal{E}_{ \pm}^{-2}\left(-A_{ \pm} d t_{ \pm}^{2}-2\left(1-A_{ \pm}\right)^{\frac{1}{2}} d t_{ \pm} d R+d R^{2}\right)+R^{2} d \Omega^{2} \\
& =-d t_{ \pm}^{2}+\mathcal{E}_{ \pm}^{-2}\left(d R-\left(1-A_{ \pm}\right)^{\frac{1}{2}} d t_{ \pm}\right)^{2}+R^{2} d \Omega^{2}
\end{aligned}
$$

where in the most general case:

$$
\begin{aligned}
& A_{+}=1-\frac{2\left(m_{+}+M_{+}\right)}{R}-\frac{\Lambda_{+}}{3} R^{2} \\
& A_{-}=1-\frac{2\left(m_{-}+M_{-}\right)}{R}-\frac{\Lambda_{-}}{3} R^{2},
\end{aligned}
$$

$m_{-}$is the Schwarzschild mass at the center, and $m_{+}=m_{-}+m_{\Sigma}$ where $m_{\Sigma}$ is the Schwarzschild mass of the shell; $\Lambda_{ \pm}$gives the vacuum energy density in $\mathcal{M}^{ \pm}$, respectively; $M_{ \pm}$are the mass functions for the Universe in $\mathcal{M}^{ \pm}$, respectively, defined in equation (19). The parameter $\mathcal{E}_{ \pm}$is given in eq. (32).

The embedding is

$$
x_{ \pm}^{\mu}=\left(t_{ \pm}(\tau), R(\tau), 0,0\right) .
$$

Thus, the 4 -velocity, $\mathbf{u}_{ \pm}$, for a comoving observer is

$$
\frac{\partial x_{ \pm}^{\mu}}{\partial \tau}=\frac{d x_{ \pm}^{\mu}}{d \tau} \equiv u^{\mu}=\left(\dot{t}_{ \pm}, \dot{R}(\tau), 0,0\right),
$$

where the dot denotes derivative with respect to the proper time $\tau$ on the shell. In terms of the intrinsic coordinates we have

$$
u^{i} \frac{\partial \phi_{ \pm}^{\alpha}}{\partial \xi^{i}}=u_{ \pm}^{\alpha}
$$

i.e. $u^{i}=(1,0,0)$.

From $\left.n^{\alpha} n_{\alpha}\right|^{ \pm}=1$ and $\left.u^{\alpha} n_{\alpha}\right|^{ \pm}=0$ using $\left.u^{\alpha} u_{\alpha}\right|^{ \pm}=-1$ we find the following covariant components for the normal vector:

$$
n_{\mu}^{ \pm}=-\zeta_{ \pm} \mathcal{E}_{ \pm}^{-1}\left(-\dot{R}, \dot{t}_{ \pm}, 0,0\right)
$$

where $\zeta= \pm 1$.

From the $h_{\tau \tau}$ component of the metric junction (90), (or from the lineelement: $d s_{ \pm}^{2}=-d \tau^{2}, \tau$ is proper time on the shell) we have:

$$
-A_{ \pm} \dot{t}_{ \pm}^{2}-2\left(1-A_{ \pm}\right)^{\frac{1}{2}} \dot{t} \dot{R}+\mathcal{E}_{ \pm}^{2}+\dot{R}^{2}=0
$$

which is a quadratic equation in $\dot{t}_{ \pm}$. Solving this gives:

$$
\dot{t}_{ \pm}=\frac{\left(\mathcal{E}_{ \pm}^{2}-A_{ \pm}\right)^{\frac{1}{2}} \dot{R}-\mathcal{E}_{ \pm} \sqrt{A_{ \pm}+\dot{R}^{2}}}{-A_{ \pm}}
$$

We make the sign choice by requiring that $\tau$ and $t_{ \pm}$are pointing in the same direction: $\dot{t}_{ \pm}>0$. Eq. (128) can be rearranged to give

$$
\dot{t}_{ \pm}=\frac{\mathcal{E}_{ \pm}^{2}+\dot{R}^{2}}{\left(1-A_{ \pm}\right)^{\frac{1}{2}} \dot{R}+\mathcal{E}_{ \pm} \sqrt{A_{ \pm}+\dot{R}^{2}}}
$$


We see that this is well behaved at the horizons $A=0$ and crossing the horizon such that $A<0$ we cannot have stationary shells, $\dot{R} \neq 0$. Also, we note here that $t_{ \pm}$cannot in general join continuously at the surface when embedding $m_{\Sigma}$ in $\mathcal{M}^{+}$. Eq. (127) also gives

$$
\frac{d R}{d t_{ \pm}}=\left(\mathcal{E}_{ \pm}^{2}-A_{ \pm}\right)^{\frac{1}{2}} \pm \mathcal{E}_{ \pm}\left(1-\dot{t}_{ \pm}^{-2}\right)^{\frac{1}{2}}=H_{ \pm} R+v_{ \pm} .
$$

From (33) we see that the first term gives the expansion of the Universe. Thus, the second term represents the velocity, $v_{ \pm}$, of the shell relative to the expansion in $\mathcal{M}^{+}$and $\mathcal{M}^{-}$, respectively. Then $\dot{t}_{ \pm}$is given by the Lorentz factor

$$
\dot{t}_{ \pm}=\frac{1}{\sqrt{1-\frac{v_{ \pm}}{\mathcal{E}_{ \pm}}}} .
$$

From eq. (94) we find the angular component of the extrinsic curvature tensor,

$$
K_{\theta \theta}=-R n^{R}=\zeta_{ \pm} R \sqrt{A_{ \pm}+\dot{R}^{2}},
$$

where we have used (126) and (128). Thus, from Lanczos eq. (101) we get the equation of motion:

$$
\zeta_{+} \sqrt{A_{+}+\dot{R}^{2}}-\zeta_{-} \sqrt{A_{-}+\dot{R}^{2}}=\frac{\mu}{R}=4 \pi R \sigma .
$$

This equation generalizes the previous junctions to include the junction for a general Universe with an embedded Schwarzschild mass.

In terms of the Gaussian normal coordinates we have $K_{\theta \theta}=-R \frac{\partial R}{\partial n}$; i.e. the sign $K_{\theta \theta}$ determines whether the radius of the surface is increasing or decreasing in the normal direction. For static spacetimes the sign of $K_{\theta \theta}$ is given by $\zeta$ and determines the spatial topology $3,8,13,32$. If either of the spacetimes are not static the sign of $K_{\theta \theta}$ does not in general give the topology; for the classification of the junction of two FRW spacetimes see [14].

Squaring (133) we find

$$
\zeta_{ \pm} \sqrt{A_{ \pm}+\dot{R}^{2}}=\frac{R}{2 \mu}\left(A_{+}-A_{-}\right) \pm \frac{\mu}{2 R}
$$

from which one can determine the sign of $K_{\theta \theta}$. Squaring (134) we get the energy equation

$$
\dot{R}^{2}=\dot{t}_{ \pm}^{2}\left(\frac{d R}{d t_{ \pm}}\right)^{2}=\frac{\mu^{2}}{4 R^{2}}-\frac{1}{2}\left(A_{+}+A_{-}\right)+\frac{R^{2}}{4 \mu^{2}}\left(A_{+}-A_{-}\right)^{2}
$$

The continuity equation (110) gives for a comoving observer:

$$
\dot{\sigma}=-2(\sigma+p) \frac{\dot{R}}{R}+\left[T_{\mu \nu} n^{\mu} u^{\nu}\right]
$$

or in terms of $\mu$ we have

$$
\dot{\mu}=-8 \pi p R \dot{R}+4 \pi R^{2}\left[T_{\mu \nu} n^{\mu} u^{\nu}\right] .
$$


The first term is due to the tangential pressure of the surface. The second term, the area of the shell times the discontinuity in the 4-momentum, can be interpreted as the mass gathered on the shell from the surroundings due to the motion of the shell relative to the cosmic fluid. The force needed to move on the surface is given by

$$
(\sigma+p) u_{i \mid l} u^{l}=-\left(\delta^{l}{ }_{i}+u_{i} u^{l}\right) p_{, l}-u_{i}\left[T_{\mu \nu} n^{\mu} u^{\nu}\right]-\left[T_{\mu \nu} n^{\mu} \frac{\partial \phi^{\nu}}{\partial \xi^{i}}\right],
$$

which for a comoving observer is zero, i.e. following the intrinsic geodesics of the surface.

The energy-momentum tensor is given in equation (15), where $V^{\mu}$ (eq. (6)) is the 4 -velocity of the cosmic fluid. Contracting with the normal $n_{\mu}$ and the 4 -velocity $u^{\mu}$ of the surface gives: $\left.V^{\mu} n_{\mu}\right|^{ \pm}=-\theta_{ \pm}\left(\left(1-A_{ \pm}\right)^{\frac{1}{2}} \dot{t}_{ \pm}-\dot{R}\right)$, and $\left.V^{\mu} u_{\mu}\right|^{ \pm}=-\dot{t}_{ \pm}$. Thus, when including the vacuum energy, the components of the energy-momentum tensor at the surface becomes:

$$
\begin{aligned}
& \left.T_{\mu \nu} n^{\mu} n^{\nu}\right|^{ \pm}=\left(\rho_{ \pm}+p_{c f}^{ \pm}\right)\left(\dot{R}-\left(1-A_{ \pm}\right)^{\frac{1}{2}} \dot{t}_{ \pm}\right)^{2}+p_{c f}^{ \pm}-\frac{\Lambda_{ \pm}}{8 \pi} \\
& \left.T_{\mu \nu} n^{\mu} u^{\nu}\right|^{ \pm}=-\zeta_{ \pm}\left(\rho_{ \pm}+p_{c f}^{ \pm}\right) \dot{t}_{ \pm}\left(\dot{R}-\left(1-A_{ \pm}\right)^{\frac{1}{2}} \dot{t}_{ \pm}\right),
\end{aligned}
$$

where $p_{c f}$ is the pressure of the cosmic fluid and $\rho$ the energy density. For a shell moving with the expansion of the Universe we have $\dot{R}=\left(\mathcal{E}^{2}-A_{ \pm}\right)^{\frac{1}{2}}$ and $\dot{t}_{ \pm}=1$. This gives $\left.T_{\mu \nu} n^{\mu} n^{\nu}\right|^{ \pm}=p_{c f}^{ \pm}-\frac{\Lambda_{ \pm}}{8 \pi}$ and $\left.T_{\mu \nu} n^{\mu} u^{\nu}\right|^{ \pm}=0$.

For an equation of state $p_{c f}=\omega \rho, M$ satisfies eq. (25). Thus, the derivative of $M$ with respect to the proper time $\tau$ on the surface may be written:

$$
\begin{aligned}
& \dot{M}_{ \pm}=\frac{\partial M_{ \pm}}{\partial t_{ \pm}}\left(\dot{t}_{ \pm}-\left(1+\omega_{ \pm}\right)\left(\mathcal{E}_{ \pm}^{2}-A_{ \pm}\right)^{-\frac{1}{2}} \dot{R}\right) \\
& \dot{M}_{ \pm}=\frac{\partial M_{ \pm}}{\partial R}\left(\dot{R}-\left(1+\omega_{ \pm}\right)\left(\mathcal{E}_{ \pm}^{2}-A_{ \pm}\right)^{\frac{1}{2}} \dot{t}_{ \pm}\right) .
\end{aligned}
$$

We will in the following restrict our discussion to $\omega=0$. We see that if the shell is moving with the expansion this is zero, $\dot{M}=0$. Also, we find that $\dot{M}>0$ for a shell expanding faster than the Universe and $\dot{M}<0$ if the shell is moving slower than the expansion of the Universe. Using eq. (142) with $\omega=0$ the normal and mixed components of the energy momentum tensor (139) and (140) can be written:

$$
\begin{aligned}
\left.T_{\mu \nu} n^{\mu} n^{\nu}\right|^{ \pm} & =\frac{\dot{M}_{ \pm}^{2}}{4 \pi R^{2} \frac{\partial M_{ \pm}}{\partial R}}-\frac{\Lambda_{ \pm}}{8 \pi} \\
\left.T_{\mu \nu} n^{\mu} u^{\nu}\right|^{ \pm} & =-\zeta_{ \pm} \frac{\dot{M}_{ \pm} \dot{t}_{ \pm}}{4 \pi R^{2}} .
\end{aligned}
$$

Furthermore, $\dot{M}$ can be written in terms of $v_{ \pm}$and $\rho_{ \pm}$by using equations (20), (25), (130) and (131):

$$
\dot{M}_{ \pm}=\frac{v_{ \pm} 4 \pi R^{2} \rho_{ \pm}}{\sqrt{1-\frac{v_{ \pm}}{\mathcal{E}_{ \pm}}}} .
$$


This gives the rate of change of the mass $\mu$,

$$
\dot{\mu}=-8 \pi p R \dot{R}-\left[\dot{t} v 4 \pi R^{2} \rho\right]
$$

where we have set $\zeta_{ \pm}=-1$. For a surface with vanishing tangential pressure, $p=0$, this has the same form as eq. 2.13 in [15].

Consider now the time component of the field equations for the surface. Combining equations (106) and (107) in the case where the shell consist of an ideal fluid (117) we find in general

$$
K_{\tau \tau}^{ \pm}=-\frac{\left[T_{\mu \nu} n^{\mu} n^{\nu}\right]}{\sigma}-\frac{2 p}{R^{2} \sigma} K_{\theta \theta}^{ \pm} \pm 4 \pi\left(\frac{1}{2} \sigma+2 p\right),
$$

where the superscripts \pm correspond to the \pm in the equation, respectively. ( $\sigma$ is the energy density of the surface). For a comoving observer $K_{\tau \tau}^{ \pm}$gives the proper (normal) acceleration, i.e. $K_{\tau \tau}^{ \pm}=a_{\mu}^{ \pm} n_{ \pm}^{\mu}$. Hence, the terms on the right in eq. (147) represent the forces on the shell making it deviate from geodesic motion in $\mathcal{M}^{+}$and $\mathcal{M}^{-}$, respectively. The first term is the difference in the normal force exerted on the shell from $\mathcal{M}^{ \pm}$, the second term is due to the tangential pressure on the surface while the last represents the self gravity of the shell.

We shall consider eq. (147) for a flat Universe with dust and vacuum energy, i.e. $p_{c f}=0$ and $\mathcal{E}=1$; also we put $\Lambda_{ \pm}=\Lambda$. Differentiating eq. (134) with respect to $\tau$, then using (128), (132), (133), (137), (142) and (143) we find

$$
K_{\tau \tau}^{ \pm}=-\zeta_{ \pm} \frac{2 p}{\sigma R} \sqrt{A_{ \pm}+\dot{R}^{2}}-\frac{1}{4 \pi R^{2} \sigma}\left[\frac{\dot{M}_{ \pm}^{2}}{\frac{\partial M_{ \pm}}{\partial R}}\right] \pm 2 \pi \sigma \pm 8 \pi p,
$$

where

$$
K_{\tau \tau}^{ \pm}=-\zeta_{ \pm} \frac{\ddot{R}+\frac{m_{ \pm}}{R^{2}}+\frac{M_{ \pm}}{R^{2}}-\frac{\Lambda_{ \pm}}{3} R+\frac{\dot{M}_{ \pm}^{2}}{R \frac{\partial M_{ \pm}}{\partial R}}}{\sqrt{1-\frac{2\left(m_{ \pm}+M_{ \pm}\right)}{R}-\frac{\Lambda_{ \pm}}{3} R^{2}+\dot{R}^{2}}} .
$$

For a test shell, $K_{\tau \tau}=0$, we recover the Friedmann eq. (35).

\section{Conclusion}

In this paper we have discussed the embedding of a Schwarzschild mass into a cosmological model using "curvature" coordinates. Extending the work by Gautreau [17] we have found approximate solutions to eq. 25] giving the mass function $\mathrm{M}$ for the Universe explicitly, and we have solved for the radial geodesics outside the embedded mass. We have considered spacetime close to $m$, where our solutions go towards the Schwarzschild spacetime. And far from $m$, our solutions approach the FLRW Universe models. In particular we have presented solutions for a flat Universe with vanishing cosmological constant and an equation of state $p=\omega \rho, \omega=$ constant. Without the embedded mass we have given solutions with and without cosmological constant for the equation of state $p=\omega \rho$.

Using the Gautreau metric we have generalized Israel's formalism to singular shells in a Sch/FLRW background. For an arbitrary equation of state for the surface the equations governing its motion are (135), (137) and (148). Equations (135) and (137) are given for the general case, while we have only considered 
the acceleration (148) for a flat pressurefree Universe model. An interesting further development would be to extend this to three-dimensional branes in a five-dimensional spacetime.

An application of the results obtained in this paper is to study the evolution of cosmological voids, see [15, 16, 33] and references therein. In 33] the collapse of a positive perturbation leading to the Einstein-Straus model is discussed, i.e. the expansion of the voids are comoving. In [15] 16] a less dense region is considered. This less dense region will expand faster than the outer region and numerical simulations show that a thin shell is formed. It would be interesting to investigate if this problem can be analyzed analytically. Also, it would be interesting to apply our formalism to slowly rotating shells, generalizing the traetments in [19 20] to shells that need not be comoving with the cosmic fluid.

\section{References}

[1] W. Israel. Singular hypersurfaces and thin shells in general relativity. Il Nuovo Cimento 44B, 1 (1966). Erratum 48B, 463 (1966).

[2] C. Barrabès and W. Israel. Thin shells in general relativity and cosmology: The lightlike limit. Phys. rev. D 43, 1129 (1991).

[3] V. A. Berezin, V. A. Kuzmin and I. I. Tkachev. Thin wall vacuum domain evolution. Phys. Lett. 120 B, 91 (1983).

[4] V. P. Frolov. The motion of charged radiating shells in the general theory of relativity and "friedmon" states. Sov. Phys. JETP, 39, 393 (1974).

[5] W. Israel. Gravitational collapse and causality. Phys. Rev., 153, 1388 (1967).

[6] V. De La Cruz and W. Israel. Gravitational bounce. Il Nuovo Cimento 51A, 744 (1967).

[7] K. Kuchař. Charged shells in general relativity. Czech Journ. Phys. B 18, 435 (1968).

[8] S. K. Blau, E. I. Guendelman and A. H. Guth. Dynamics of false-vacuum bubbles. Phys. rev. D 35, 1747 (1987).

[9] A. Corichi, G. Cruz, A. Minzoni, P. Padilla, M. Rosenbaum, M. P. Ryan Jr, N.F. Smyth and T. Vukasinac. Quantum Collapse of a small dust shell. gr-qc/0109057, (2001).

[10] V. A. Berezin. Quantum black hole model and Hawking's radiation. Phys. rev. D 55, 2139 (1997).

[11] D. Langlois. Brane cosmology: an introduction. In Braneworld -Dynamics of spacetime boundary, 2002. hep-th/0209261.

[12] T. Shiromizu, K. Maeda and M. Sasaki. The Einstein equations on the 3-brane world. Phys. rev. D , 62, 024012 (2000).

[13] V. A. Berezin, V. A. Kuzmin and I. I. Tkachev. Dynamics of bubbles in general relativity. Phys. rev. D 36, 2919 (1987). 
[14] N. Sakai and K. Maeda. Junction conditions of Friedmann-RobertsonWalker space-times. Phys. rev. D 50, 5425 (1994). gr-qc/9311024 v2 (1994)

[15] N. Sakai, K. Maeda and H. Sato. Expanding shell around a void and Universe model. Progress of Theoretical Physics 89, 1193 (1993).

[16] K. Maeda and H. Sato. Expansion of a thin shell around a void in expanding Universe. Progress of Theoretical Physics 70, 772 (1983).

[17] R. Gautreau. Curvature coordinates in cosmology. Phys. Rev. D 29, 186 (1984). Imbedding a Schwarzschild mass into cosmology. Phys. Rev. D 29, 198 (1984).

[18] N. Van den Bergh and P. Wils. Imbedding a Schwarzschild mass into cosmology. Phys. Rev D 29, 3002 (1984).

[19] C. Klein. Rotational perturbations and frame dragging in a Friedmann universe. Class. Quantum. Grav. 10, 1619 (1993).

[20] T. Doležel, J. Bičák and N. Deruelle Slowly rotating voids in cosmology. Class. Quantum. Grav. 17, 2719 (2000).

[21] R. Gautreau. Newton's time and space in general relativity. Am. J. Phys. 68, 350 (2000).

[22] R. Gautreau. Geodesic coordinates in the de-Sitter Universe. Phys. Rev. D 27, 764 (1983).

[23] E. Eriksen and Ø. Grøn. The de-Sitter Universe model. Int. J. Mod. Phys. $\mathrm{D}, \mathbf{4}, 115$ (1995).

[24] A. Einstein and E. G. Straus. Rev. Mod. Phys. 17, 120 (1945). Rev. Mod. Phys. 18, 148 (1946).

[25] E. Schücking. Z. Phys. 137, 595 (1954).

[26] W. B. Bonnor. Class. Quantum Grav. 17, 2739 (2000).

[27] M. Mars. Class. Quantum Grav. 18, 3645 (2001).

[28] Ø. Grøn. Repulsive gravitation and inflationary Universe models. Am. J. Phys. 54, 46 (1986).

[29] R. Gautreau. Cosmological Schwarzschild radii and Newtonian gravitational theory. Am. J. Phys. 64, 1457 (1996).

[30] Ø. Grøn. A new standard model of the Universe. Eur. J. Phys. 23, 135 (2002).

[31] C. J. S. Clark and T. Dray. Junction conditions for null hypersurfaces. Class. Quantum Grav. 4, 265 (1987).

[32] H. Sato. Motion of a shell at metric junction. Prog. Theo. Phys. 76, 1250 (1986).

[33] C. Stornaido. Cosmological black holes. General relativity and gravitation 34, 2089 (2002). 